\title{
Deconstructing Silos:
}

\section{The Business Value of Usability in the $21^{\text {st }}$ Century}

\author{
Gitte Lindgaard \\ Carleton Human Computer Interaction Institute \\ Carleton University \\ Ottawa, Ontario, Canada \\ gitte lindgaard@.carleton.ca
}

\begin{abstract}
This talk aims to show how traditional divisions of labour and responsibilities prevent businesses from adopting a customer focus, and, more importantly, the negative impact this has on their bottom line. I discuss how Human Computer Interaction $(\mathrm{HCl})$ specialists can help to break down this silo structure and establish a user- or customer-centred focus. By applying $\mathrm{HCI}$ methods wisely, internal communication patterns can be revised to maximise the business value of a User-Centred Design (UCD) approach. Focusing first on the Systems Design \& Development Process, I draw attention to certain points at which $\mathrm{HCI}$ can easily be integrated into the process, outlining some of the costs and the benefits an individual IT project stands to gain. Invariably, both of these sets of figures are surprisingly high. A brief discussion of the user- versus the customer experience aims to show their similarities and how they differ.
\end{abstract}

Key words: ROI, usability, task analysis, stakeholder

\section{INTRODUCTION}

Imagine a scenario like the following. Michael S, a 34-year old telco sales manager, has just installed a new super printer. Having tried every trick he knows, and having read all 189 pages of mostly uninterpretable instructions in the user manual, the printer still refuses to cooperate. Michael cannot get it to print. A thorough search finally leads him to the telephone number of 'Customer Support' on page 153 in the user manual, so he calls the company. Operator: "Good afternoon. This is SuperStarX. My name is Petra. Can I help you?" Customer: "Oh, excuse me, is this BestPrinter 
customer services?". "Yes it is, Sir. What can I do for you?". "Well, I bought a printer this morning. I have just installed it but it refuses to print." "Have you sent in the warranty card yet?" "No. I have only just unpacked the printer." "Ok, strictly speaking I am not allowed to help you until the warranty procedures have been completed, but seeing that you are on the line I will do so anyway. What version is your printer?" "Oh, I think it is called a CXP1800S or SX, but I am not sure. It doesn't say on the printer. It is a $\mathrm{B} \& \mathrm{~W}$ laser printer. I have the user manual in front of me, though. It lists a whole range of printers, but there are no pictures, so I can't really tell." "Mmmm! what you are telling me can't be right. I will need you to turn the printer upside down and look in the far right corner. There should be a name just above the serial number."..... etc. etc. etc, until, after a 15-minute question-answer game in which the friendly operator works tirelessly and patiently to establish the source of the problem finally succeeds, saying something like "I am terribly sorry, Sir, but I can't help you with that. Yours is a technical problem. You will need to call Tech Support. Their number is 7891015566 . Thank you for calling SuperStarX." .... Or, if the company is really customer-centred: "I am terribly sorry, Sir, but I can't help you with that. Yours is a technical problem. I will try to connect you to a Tech Support agent, but in case I lose you, their number is 7891015566 . Thank you for calling SuperStarX. Please stay on the line while I connect you".

"So what?", I hear you think. "What has all this got to do with the IT Department?" After all, Customer Support, Help Desk, and Tech Support are not owned by the Product Development, the IT Section, the Systems Branch, or whatever the development arm is called in your company - it falls squarely under the auspices of Operations. Seen from inside a large organisation, yes, the problem of serving customers belongs to someone else. But think for a moment of yourself as wearing the customer's hat: in that role it does not matter to you who inside 'owns' 'your' problem. What does matter is that it be fixed as quickly, as effortlessly, and with as little fuss as possible. Unfortunately, companies all too often send customers on a wild goose chase from pillar to post to solve a problem that should not have occurred in the first place. Even worse, too often companies do not know (a) that this particular problem exists, (b) how frequently it occurs, (c) how much it is costing in customer service, or (d) how to extract the business value from customer feedback, including the calls customers make to the company's own help desk.

In this talk I aim to show how traditional divisions of labour and responsibilities prevent the business from adopting a customer focus, and, more importantly, the negative impact this has on its bottom line. I discuss how Human Computer Interaction (HCI) specialists can help to break down this silo structure and establish a user- or customer-centred focus. By 
applying $\mathrm{HCI}$ methods wisely, internal communication patterns can be revised to maximise the business value of a User Centred Design (UCD) approach. Focusing first on the Systems Design \& Development Process, I draw attention to certain points at which $\mathrm{HCI}$ can and should be integrated into the process, outlining some of the costs and benefits an individual IT project stands to gain. A brief discussion of the user- versus the customer experience aims to show their similarities and how they differ. The main difference, I argue, is in the relative size of the 'experience' envelope. But first - what exactly are the problems with the above scenario?

\section{INTERNAL COMMUNICATION BARRIERS: ORGANISATIONAL SILOS}

In a typical organisation, a satisfactory solution to the problems in the above example could involve at least six different departments, depending on where the problem source is. Hardware designers decide where to place different kinds of information, including the name and version of the product. The ideal place is certainly not on the bottom of a product that should never be turned upside down once the print cartridge has been installed. Software designers are responsible for ensuring the correctness, completeness, and usability of the installation procedure, but Michael may have been unable to understand what to do from the information provided on the screen. Quality Assurance takes ownership of delivering fault free products, but it is possible that some aspect of the product was faulty. Technical writers own the user manual, but the instructions provided in the manual may have been unclear. At any rate, they were apparently well hidden, as indeed was the help desk telephone number. The training department ensures that help desk operators are equipped to diagnose and solve customers' problems efficiently. It is unclear whether Michael's problem was pointing to inadequate operator training or whether internal turf protection simply got in the way of providing the operator with the information necessary to solve the problem. Finally, customer services are responsible for help desk operations. It may have failed to notify the training department of the need to train operators adequately to deal with this particular problem as well as in the effective use of the telephone. If the company has developed its own help desk support system, the IT department may be responsible for providing correct and complete information for the help desk operator. Evidently, the operator's system was of little help in diagnosing, and of no help in solving the problem. The important lesson here is to note that no one department could take ownership of Michael's problem, and without ownership it is unlikely to be resolved. The customer 
service as a complete package could certainly do with a serious overhaul. To put it bluntly - the customer experience here sucks!

Because it is so difficult for people to communicate across the boundaries of departments in typical companies, it is unlikely that the information about Michael's inability to locate the printer name would ever get back to the hardware designers. Likewise, the inconvenience to the user of hiding the help desk telephone number towards the back of the user manual is unlikely ever to reach the technical writers. But without this feedback, how are hardware designers supposed to fix the problem or even to know what problem(s) to fix? How are technical writers expected to improve their documentation? Indeed, how is the business going to improve its products and services, and how can it possibly monitor progress without mining and integrating feedback from customers into all its business processes? Sales figures alone provide only one source of feedback, and no company can afford to release products into the market place with sales projections based on no firm data whatsoever. However, few of the data sources that ARE available and could assist in making business predictions are utilized to the full. This, I claim, is one area in which $\mathrm{HCl}$ experts can help.

One important factor in dysfunctional customer experiences is thus the way large organisations tend to be structured, in silos. Each department has its own turf, its well-defined responsibilities, budgets, boundaries, and accountabilities - it lives in its own silo. However, across the board, no one "owns" the customer experience as a whole in such organisations and so, no resources are devoted to tracking precisely that. Everyone owns one perspective and each of these represents a mere fraction of the total customer experience. Not surprisingly, efforts to repair it tend to amount to uncoordinated band aid treatment, with each department seeking to improve its contribution in isolation, often in a vacuum completely devoid of feedback from actual customers or from other departments. It is not a lack of organisational motivation that underlies a fractured customer experience. Each department is usually trying its very best to serve customers well from its own limited view of the world, but in the absence of a company wide coordinated customer focus the customer experience is likely to be confused and fragmented.

One major reason why companies have not yet embraced a customercentred focus is that both the costs associated with poor, uncoordinated customer service, and the benefits that could accrue from adopting a holistic business perspective are largely invisible. However, case studies that outline costs and benefits associated with customers usually report such staggering figures that one has to admit that, as a profession, we have not yet communicated our message effectively. For example, in one case users had problems installing a printer driver, resulting in more than 50,000 calls to the 
help desk, the services of which amounted to roughly $\$ 500,000$ per month, or a cool $\$ 6$ million per year. The estimated cost of a help desk call lies between $\$ 12$ and $\$ 250$ (Wiklund, 1994). Translated into the printer driver problem, additional costs thus amounted to somewhere between $\$ 600,000$ and $\$ 12,5$ million. Apart from this cost, the manufacturer sent out letters of apology to all customers with a patch diskette costing $\$ 3$ each, spending some $\$ 900,000$ on fixing this simple, small problem (Donahue, 2001). In another case, the mere design and implementation of an internal documentation style guide and template in a large telecommunications company saved $50 \%$ of the documentation production costs which, at the time, amounted to roughly $\$ 140,000$ per month, or $\$ 1.68$ million per year. The cost of producing these tools was $\$ 40,000$. The payback period was thus a mere 17 days (Lindgaard, 1995). Availability of the tools relieved the technical writers of many low level design decisions normally made for each individual document. Likewise, time-consuming negotiations with clients who owned the products for which the user manuals were being written were rendered unnecessary, saving valuable client time as well that was not included in the equation. Over time, the consistency across user manuals that the tools achieved helped to increase customer satisfaction for the business as a whole. Customers simply learned the 'geography' of the user manuals so that they knew what information to expect approximately where in any user documentation.

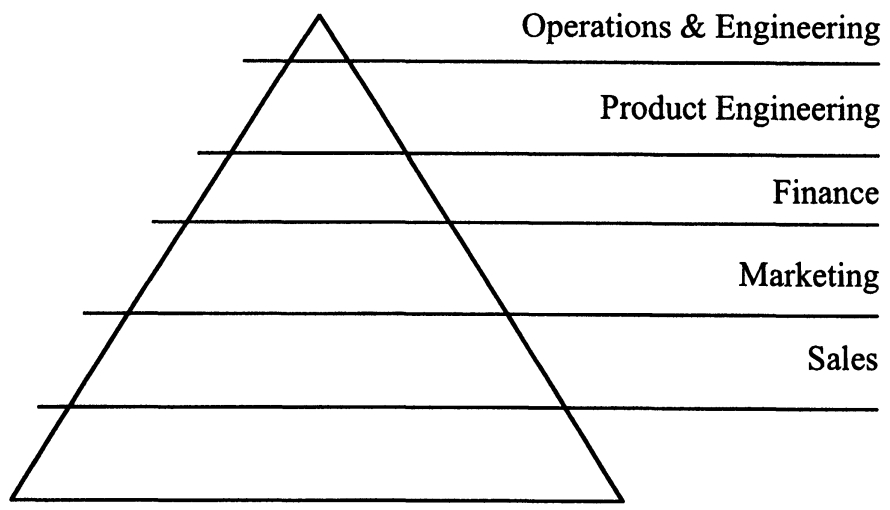

Figure 1: The spread of information in a silo-structured organisation

In order to focus on the end-to-end customer experience, we need to collect and coordinate data obtained from a variety of sources. A study just completed in our lab showed that knowledge of a high proportion of questions customers asked of the help desk personnel could benefit several 
departments. However, the information stayed in the help desk system, which was used to keep track of the status of problems until they had been resolved. Valuable customer feedback was thus lost to the business. For example, it is extremely useful for the CIO to know that, say, $5 \%$ of customers of a particular product have problems using a certain feature. The problem can then be resolved in the next run and avoided in all related products in the future.

It is virtually impossible to cross traditional organisational boundaries that characterise the silo model. In one part of a large case study aiming to facilitate access to large amounts of data (Fabris, 1999), the author asked 70 people to describe from whom they received documents and to whom they sent them. This information enabled him to track information flow through the company and hence to model the internal communication patterns and structure. He learned that company politics determined who gets to see what information when in the product life cycle. Secrecy was jealously guarded principally to keep competitors guessing the company's strategy. He learned that most projects followed a pattern in which the earliest stages were the most secretive. The conception and early development phases were driven by operations and engineering executives. Thereafter, product engineers would start the design. Eventually, finance, marketing, public relations and the sales departments would get involved. This way, as illustrated in Figure 1 , more people became involved and saw the specifications after each milestone was reached. Access to information was contained strictly within projects so that a manager heading one project had no access to information about other ongoing projects.

Not only does this model prevent transfer of learning between concurrent projects, it also blocks learning between project teams over time as well as being a hindrance to incremental organisational learning. People do not have an opportunity to learn tips, tricks and pitfalls from each other, and errors occurring in one project are likely to raise their head in others. Consistency between products is extremely difficult to achieve under these circumstances, and it is impossible for a project to benefit from solutions developed in another. As a consequence, the proverbial wheel may well be reinvented many times over. To the customer buying, or thinking of buying, a company's products, there is little chance for transfer of learning from one generation of the product to the next, especially if the two were developed by different project teams. This is unimportant for many consumer products such as hair dryers or washing machines that perform basic and well-known functions, but it does become an issue when dealing with more complex products such as PDAs, photocopiers, or printers for that matter. 


\subsection{Integrating usability into systems development}

In a recent paper, Rubin (2002) discusses what he calls the 'three waves of usability'. Rubin attributes the first wave to Human Factors research aiming to improve airplane cockpit design during the Second World War. Until then, knobs and dials were placed wherever they would fit in the very restricted environment without regard for the pilot, his physical and cognitive capabilities or limitations. The second usability wave, Rubin attributes to the arrival of computers, from mainframes to the later ubiquitous Personal Computer. During this second wave, Rubin argues, the objective was to improve the performance of computer users. Improvements were achieved largely through empirical testing of prototypes, an understanding of tasks and task flows, but there was no talk about the customer experience as a whole. He goes on to argue that the third usability wave focuses on the wider customer experience which expands beyond the sheer interaction between an individual user and a computer terminal to encompass the end-to-end interaction with a company.

It is true that the Internet is assuming an increasingly important role in most people's lives in the western world and that therefore, the end-to-end customer experience has risen in importance. However, Rubin's 'second usability wave' is still highly relevant to the design, development, implementation, use, and evaluation of traditional applications, many of which are developed for internal use. Furthermore, web-based Intranets and Extranets increasingly resemble the custom-tailored applications that evolved from the text-based systems - to GUI applications in the late eighties and early nineties for which we designed style guides and guidelines, and in the context of which usability evaluation methods emerged from a range of disciplines. While the application development time frames have been reduced dramatically compared with those of the mid-nineties, the need for usable and useful applications has not diminished. Quite the contrary! With increasing experience users have become much more discerning, more demanding and less tolerant of unforgiving, uncompromising and unusable applications. In the context of business-to-customer e-commerce, potential customers are likely to move on to the next company if they encounter usability problems.

Enough is known about how to achieve high levels of usability that there is no excuse for releasing cumbersome applications or web sites. Yet, according to some researchers, a sobering $65 \%$ of potential online shoppers give up before completing a transaction (Souza, 2001). At the same time, ease of use is seen as the most important element in web site design (Souza, 2001). However, a survey of Fortune 1000 companies showed that these spend an average of $\$ 1.5$ million to $\$ 2.1$ million per year on site redesigns 
without knowing whether the redesign makes the site easier to use (Kalin, 1999). One widespread misunderstanding is that usability is 'soft' and cannot be measured. Therefore, many companies set 'soft' redesign goals that tell them precisely nothing about Return Of Investment (ROI). A goal, for example, to "improve the site", "update the look and feel", or "make the site simpler" (Souza, 2001) is useless, impossible to measure, and holds nobody accountable. Without quantifiable usability and business goals there is no way of knowing if usability improvements have been achieved. It is much like saying that "a business must make a profit". HCI does have methods, processes, and metrics that are readily integrated into the systems design and development lifecycle (Lindgaard, 1994; Vredenburg et al., 2001). Let us therefore look at a number of ways that $\mathrm{HCI}$ can contribute to improving applications.

\subsubsection{Usability in the requirements capture phase}

Software engineers have long known that if the cost of fixing a problem during the requirements capture phase is one unit, this increases to 10 units if fixed during the development phase and to $100+$ if coming to the fore after an application has been released (Pressman, 1992). Likewise, it is well known that $80 \%$ of service costs are spent on unforeseen user requirements that have been neglected during the design phase (Karat, 1997). Indeed, $80 \%$ of software problems only emerge once the application has been released, and of these, $80 \%$ could have been avoided if a complete User Needs Analysis (UNA) (Lindgaard, 1994) had been performed during requirements capture. In outlining a set of HCI methods and findings in the discussion below I will assume redesign of an existing application for internal use.

$\mathrm{HCI}$ objectives during the requirements capture phase are first to facilitate a shared and realistic vision for the application among all stakeholders. Second, task analysis facilitates a detailed understanding of the tasks users perform. Task analysis highlights problems with the task procedure and the tools, it yields a concrete basis for setting usability goals, and it helps to identify how and where improvements may be made to all of these. Knowledge gleaned from the task analysis forms the basis for designing future tasks, for shaping the future tool/application, and for predicting future task performance.

\subsubsection{Stakeholder analysis}

The objective of the stakeholder analysis is to develop a shared vision of the future application and to agree on the role it will fulfil as well as decide on the features it will contain. If marketing wants to promote certain features in the future product, IT needs to assess the technical feasibility of including 
those features in the planned software version, and whether it has the expertise to make firm promises given the restricted time lines and budget it works under. Finance must be involved to ensure that the cost of the features will be covered. The training department needs to assess the cost of its commitment to train users. If the product is intended for clients outside the organisation, questions such as how much training will add to the customers' expense need to be asked. How these features relate to the company's strategic plans, the life cycle of the product, and its relationship with other, similar products in its range are also issues to be discussed, as is the business goal with the product - is the company to be first to bring the product to market? Is it intended to increase market share? To enter into a new market sector? Or are the features to be provided because the competitors are bringing out the same features?

No doubt compromises will need to be made - give a little here, take a little there, but bringing all the stakeholders together to discuss and eventually agree on the business goal helps everyone to set realistic and achievable goals. The stakeholder analysis follows a structured approach with a number of questions to be answered (e.g. Maguire, 1997).

\subsubsection{Task analysis/task projection}

Deriving a detailed understanding of the users' tasks is seen by many as a waste of time because, it is argued, "we are designing for the future here - no point recreating the present". True, but what this viewpoint misses is that without knowing how, why, when, and where the present application fails to support the users' tasks, there is little chance of eliminating those stumbling blocks. Similarly, rather than redesigning the way these tasks will be performed in a vacuum, it makes sense to understand what works well and what causes problems for users.

Alternatively, it is often believed that 'someone', for example, the business analysts, systems analysts, marketing, sales personnel, or whoever already has that information at hand. Not so! The level of detail we need to improve the processes and tasks to be supported is not contained in any other document or indeed in anyone's head. If it were we would not find that $80 \%$ of problems revealed after release point to problems in the requirements capture phase. In one case, in which a task analysis was performed the signon procedure in an application used by several thousand people was found to be more cumbersome than necessary. The company spent $\$ 20,700$ on usability work to improve it. The resulting improvement saved the company $\$ 41,700$ on the first day the new procedure was used (Karat, 1990).

In a task analysis of telco customer service operators mentioned earlier my research team found that each operator spent 85 minutes per day on unnecessary activities during customer calls. Activities labeled unnecessary 
were those for which the information needed in the transaction should have been displayed on the screen but were not, and where the customer was put hold during the transaction to obtain the information. For example, the need to call the organisation 18 times a day to book a technician to visit a customer could have been avoided if operators had had access to the technicians' schedules. The communication bill to cover this activity alone amounted to $\$ 1.6$ million per annum, not including the 34 seconds these calls took on average, involving both the operator and another person in the organisation.

Of these 85 minutes wasted per day per operator, over half could be reduced dramatically if not entirely eliminated with relatively little effort on behalf of the project team, by simply providing the needed information during the transaction. Since these figures provide valuable information that can be employed in project usability goals, it is worth discussing just one of these activities in some detail. Let me focus on one such activity occurring when connecting a new line. The operator must find and allocate a phone number. Vacant numbers were kept in a hardcopy "Vacant Number File" (VNF) floating around somewhere in the open-plan office shared by some 35 operators. The customer was put on hold while the operator located the VNF which was never in the place it was supposed to be kept; operators tended to bring it to their desk so as to cross out the number they had just selected. Once the VNF had been found, the operator would write down several numbers in her hand and race back to her desk. Next, she would try ringing the first number on her list to ensure it had not already taken, in which case she would hear a ringing tone. If the number had been taken, she would try the next on her list and so on until a vacant line could be found. At this point she would pick up the customer again, enter the number in her file and complete the transaction. Upon completion of the call, the operator was expected to locate the VNF again and cross out the number she had just allocated. Having timed several hundred calls in this category, an event diagram was produced showing the average time spent on each activity including this one in the average 12-minute call. No one knew that this or any of the many other problems revealed by the study existed in the six types of transaction these operators performed. Clearly, the solution was to present the VNF online and remove phone numbers as they were taken, a mere cut and paste operation which should take maximum 5 seconds. Even allowing a generous 10 seconds for the operator to call up the list and select a number, this still represented a saving of 4 minutes and 20 seconds per transaction (Lindgaard, 1992). With an average of six such transactions per day per operator and a total of 2,500 operators across the country earning an average of $\$ 25,000$ per annum, the cost of the VNF procedure is easy to calculate: 


\section{Operator costs before redesign:}

Annual salary per operator per year $\quad \$ \quad 25,000.00$

Salary per day, counting 215 working days per annum $\$ \quad 116.28$

Salary per minute, counting 450 working minutes per day $\$ \quad 0.26$

Salary per call @ 4 minutes 30 seconds $\quad \$ \quad 1.16$

Salary for 6 calls per day per operator $\quad \$ \quad 6.97$

Salary for 6 calls/day and 215 days per operator $\quad \$ \quad 1,496.40$

and for 2,500 operators per year $\quad \$ \$ 3,741,000.00$

\section{Operator costs after redesign:}

Salary for 10 seconds per call per operator per year $\quad \$ \quad 55.90$

and for 2,500 operators per year

\section{Savings:}

Costs before redesign

$\$ 3,741,000.00$

less costs after redesign

$\$ 139749.89$

Total savings per year

Thus, merely by making the VNF available online, the company would save over $\$ 3$ million per year, and this was not the only place or the only transaction in which savings could be made with comparatively little effort (Lindgaard, 1992).

The important point to note is that, in addition to the sheer dollar value for the business, figures such as these also feed directly into setting quantifiable usability goals. In this transaction and from the above data alone, our data clearly showed that 4 minutes and 20 seconds would be saved per call, or 93 hours 10 minutes per operator per year - more than two full working weeks! Even if nothing else were changed in the user interface or in the way the transaction was to be performed in the redesign, this saving was a concrete, risk-free business goal that the project team knew it could make by letting the task analysis data tell their story. The trick is, of course, to know what transactions are to be supported in the new application and what activities these involve. It is impossible and meaningless to measure everything an operator does. Therefore, the stakeholder analysis helps to focus on those tasks that will continue to be supported and in which it quickly becomes obvious that business value is to be gained relatively easily.

\subsubsection{Shortcomings of the existing application}

Ok, so task analysis data should help the analyst identify how and where in the transactions there is scope for tangible, quantifiable improvements. The application itself has, however, not been absolved yet. An $\mathrm{HCI}$ audit 
involves a thorough going over of all screens and transactions using standards and guidelines for information design as well as observing users in action to identify stumbling blocks. Information \& Screen Design Standards are based on principles of human information processing, psychophysics, human memory, social norms and other human capabilities and limitations. Our research on telco operators revealed that the screen design principles employed in the application were highly problematic. When interacting with a customer on the phone, for example, operators tend to request personal details in a certain order, following the social norms of their particular culture. Upon requesting their surname, they naturally ask for a first name, then initial. Next comes the address - Street number, Street name, Suburb or Town, and so on. Our observations showed that operators invariably followed these social norms instead of the form layout on the screen. This forced them to jump all over the screen, as data belonging together were separated in what seemed a random fashion. Apart from rendering the interaction cumbersome, this also had a severe impact on the accuracy of operator performance. One in every five operations had to be repeated to locate a missing entry,which added significantly to the transaction time. However, the application refused to accept an incomplete transaction, forcing the operator to walk through up to 15 screens to find the culprit. Even so, $30 \%$ of transactions accepted by the application were later refused, causing a delay in the delivery of whatever service the customer had requested as well as providing full time work to another 35 people elsewhere in the organisation. As with the previous example, many of the problems could easily be rectified by rearranging the data entry forms in accordance with social norms and telephone manners.

Again, simply by observing how much the design blocks the interaction and by applying a model much as described above it is possible and easy to estimate the cost of these as well as the value of improvements. Likewise, these observations help to identify benchmark tasks on which to assess the magnitude of improvements in the redesign. In that sense, the field observations provide baseline data against which usability goals are set and measured in the redesign.

\subsubsection{Usability goals, benchmark tasks, and task scenarios}

Armed with analyses of the critical tasks and realistic improvement indicators to be employed in the redesign, the stakeholders can now meet again to set usability goals and select benchmark tasks. Usability goals are concrete and quantifiable, stated in business terms, and they are based on the observations made in the task analysis and application audit. Tasks that are critical for the business are likely to be known already, in which case much of the task analysis effort concentrates on collecting data that point to 
opportunities for improvements to these. The task analysis may reveal frequently performed, but problematic, tasks with severe stumbling blocks that should be included in the benchmarks as well.

The purpose of the stakeholder discussion at this point in the procedure is to agree on a set of realistic usability goals and the level of usability that should be attained. For example, 'connecting a new line' was a critical task. The usability goal could be expressed as "At least $95 \%$ of test users must be able to work through the transaction in less than five minutes, committing maximum one error. If an error is committed, the operator must be able to recover in a single attempt." This criterion may turn out to be too ambitious, so it is set as the 'ideal'. A lower criterion, say $90 \%$ of test users and allowing two errors and two trials to recover, may represent an 'acceptable' goal, and finally a yet lower one may determine the 'minimum acceptable' level of usability for the application to 'pass' the final usability test on this task. Because a shared vision has evolved for the product, and since the usability goals are set in accordance with business goals and based on actual data, the risk of failing to attain them is very low.

Task scenarios are stories that serve to guide both the user interface designers and usability test users by providing a conceptual hatrack. Scenarios are written for all the benchmark tasks, and users from the field help us write them to ensure face validity and that no details have been left out. A task scenario provides an end-to-end detailed walkthrough of the task. When designing the user interface, scenarios enable the designers to check that all information appears in the right place and the right time in the transaction, and in the format that best suits the particular users for whom the application is designed. In the usability test, it provides all data the user will need to perform the task.

All the facts, figures, observations, business and usability goals, scenarios, and benchmark tasks are now stored in the stakeholder report, which is signed off by the key stakeholders.

\subsubsection{High-level user interface design}

As every good software engineer knows, testing is done just before the application is shipped. Not so in HCI! Not surprisingly, this cultural difference causes a lot of irritation at times - none of us would like some smart charlies to tell us how to do our expert job, especially if they tell us to change our ways. In our $\mathrm{HCI}$ world, the earlier we test the emerging user interface, the more problems we can iron out before coding even begins. So, delaying tests till the end is bad, bad news for us! Therefore, as we approach the end of the UNA we design the user interface at a high level to accommodate at least the benchmark tasks. The very first user interface draft is actually a series of drafts. In this early phase the objective is to generate as 
many presentation ideas as possible in rapid succession and without critique. Next, we sort the wheat from the chaff, throwing out the least appealing examples and those that will not work, and we hang on to the best features of the sample just generated. Eventually, we select the design we want to take further. This draft, still in paper form, is then expanded to accommodate the benchmark tasks. Believe it or not, but testing is actually very valuable at this point, before the next version is created using standard rapid prototyping tools, even just Visual Basic or PowerPoint. This cycle of design, test, revision, test is continued until it is evident that the usability goals can be reached and the design does not overstep the boundaries of the technology to be employed. This is the point at which coding the user interface can begin.

\subsubsection{The development phase}

Testing continues during the development phase, according to the Test \& Evaluation Plan and as modules become available. The skilled HCI specialist will have designed the tests so that data obtained in one test can be directly compared with those arising from the next. That is, the measures and the conditions under which they are collected are held constant. It is this consistency between tests that enables us to map our progress towards accomplishing the usability goals. If it turns out that even the 'minimum acceptable' usability goal cannot be accomplished for a given task, the stakeholders are called back as soon as this is obvious. The goals are then either renegotiated or more resources are devoted to the project, depending on the flexibility of the business goals.

In this phase the HCI folks coordinate their activities with the technical writers and the training people to ensure consistency throughout. Just like usability goals are set for the application proper, so goals are also set for documentation usability and for the adequacy of the evolving training program.

The implementation, or roll-out, plan is now designed, and the impact on users can be assessed more accurately. This activity is performed together with human resources, and with the Occupational Health \& Safety personnel to ensure the job design is updated and acceptable to users and their union. It is also necessary to estimate the time for the system to be 'run in' and to allocate additional resources in the work place during this period. Unfortunately, it is often the case in Anglo-Saxon and Anglo-American companies that massive savings achieved with a new application in terms of staff time are translated into down-sizing the staff. Contrast that with the large Swedish insurance company that, upon streamlining its internal computer systems, entrusted the front line operators with client portfolios to ascertain how the company could save its customers money by rearranging 
their existing insurance, going through these one by one (Edvinsson \& Malone, 1997). Rather than dismissing the people who could now have been regarded as superfluous, the company maximised the value of its human capital by adopting this pro-active procedure. Human capital is defined as the "sum total of all knowledge, experience and human performance capability on organisation processes that can be applied to create wealth" (Stolovitch \& Maurice, 2001). Not only did the change in operator jobs in the Scandinavian company result in higher satisfaction levels among the staff, it also increased the business by several hundred percent in the first two years.

\subsubsection{The implementation and 'in use' phase}

It makes sense for HCI people to 'hang out' in the work place during implementation of the application simply because a lot can be learned about what is going well and what is amiss immediately after the system has been implemented. Special attention is paid to issues like what must be done differently next time, what can be avoided, and how the process can be handled better.

Post-implementation field studies are invaluable for collecting the kinds of data I outlined in the requirements capture phase, but field studies are unfortunately rarely performed. New projects come along, resources are scarce, life moves on. However, if performance were monitored even through regular spot checks, much time would be saved in the planning and requirements capture phase of the next version of the same or a similar application.

\subsection{User experience vis à vis customer experience}

For those of you who are involved in developing consumer products, web sites and the like, I hear your sighs. Yes, so far I have talked about the development of applications for internal use. However, the procedures involved in these are not so different from those in which we design for consumers. The relationship between these is shown in Figure 2 .

The point of the Figure is to show how the customer experience differs from that of internal employees. Customers see the product later in its lifecycle, but employees are involved throughout the development as well.

The lower part of the Figure shows the feedback loops in a typical consumer product. During development, the main phases of which are shown in the enclosed large box, information flows between the IT and other departments in iterative cycles. The IT department as such is unlikely to be directly involved in feasibility studies, but information from these flows into 
it. Likewise, IT usually has little say in the product lifecycle in terms of when it is phased out. This is driven partly by the strategic business goals and partly by customers.

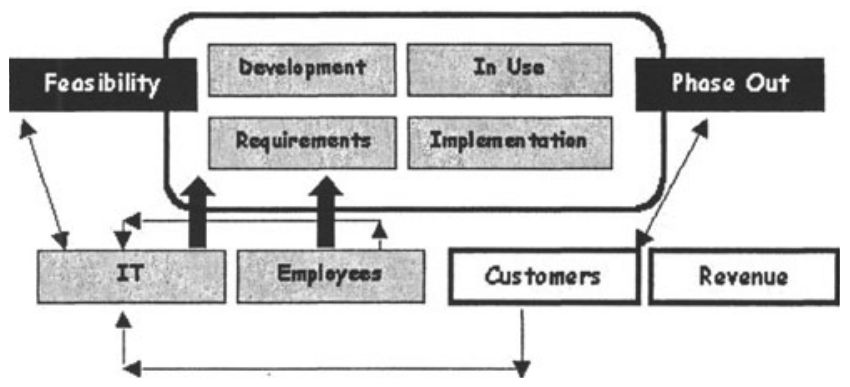

Figure 2: The relationship between IT design/development processes and the consumer business model

Employees usually have little say in whether or not a given product is purchased, developed, redeveloped or phased out, but customers must be persuaded to buy your company's products instead of selecting competitors' wares. The entire marketing and sales machinery comes into play, and issues from packaging to advertising, from usability to aesthetic appeal and your company's reputation all play a role in the success of the product. Thus, the 'customer experience' envelope is much broader than that of the internal user. Space does not allow a detailed discussion of the customer experience here. Suffice it to say that, although it offers many more opportunities for success or failure than the internal product or application, the role of the latter should not be disregarded. Flaws in the tools, tasks, or in the environment in which employees work can be extremely costly in both human and ROI terms. By focusing on users, $\mathrm{HCI}$ offers methods, tools and techniques that can improve both substantially.

\subsection{The cost of usability}

Oh, I hear you say, but all this usability stuff delays our development schedules. HCI folks impose a lot more work on us, this soft fluff is impossible to incorporate into our development methodology, and they cost a lot of money too. No, no, and no! Earlier I alluded with a glimpse to just one example of the benefits a thorough UNA can bring. In that same study, the total cost to the business of the various flaws to the system, its 
procedures, and the impact on work processes around the business amounted to an annual loss of $\$ 30$ million. The substantiating data showed clearly that nearly half of this loss could be avoided before the application was even touched (Lindgaard, 1995). The HCI costs of this comprehensive ergonomic review were $\$ 90,000$. The user-centred approach takes into consideration the wider business context rather than focusing exclusively on the application. Yet, it benefits both. It is estimated that the return for every dollar invested in usability is somewhere between $\$ 10$ and $\$ 100$ and that a lack of usability input to the design translates into $80 \%$ of service costs on unforeseen user requirements further down the line (IBM, 2001). Evidence supporting these figures is mounting in the literature.

\subsection{Silo replacements}

The model shown in Figure 2 suggests that information is flowing freely between 'employees', a group that amorphously includes everyone in the organisation, and the IT department. Unfortunately, that is usually not the case - companies are still predominantly organised into silos. The biggest hindrance for a business to become truly customer centred is this traditional parallel positioning of people who will never know how much they have in common or how much sharing information could benefit their jobs and the business as a whole. Our reward structures are designed to reward individuals for contributions that amount to fragments of the customer experience rather than rewarding whole teams who take collective responsibility for the success of the company's products and the end-to-end customer experience. Breaking down these barriers to free up communication lines and allowing people access to information that focuses on the higher level business goals while keeping an eye on the details is the only way business can evolve into becoming user and customer-centred. This paper is an attempt to demonstrate how HCI folks can help achieve this goal.

\section{REFERENCES}

Donahue, G.M. (2001). Usability and the Bottom Line, IEEE Software, January/February.

Edvinsson, L. \& Malone, M.S. (1997). Intellectual capital: Realizing your company's true value by finding its hidden brain power, HarperBusiness, New York, N.Y.

Fabris, P. (1999). You think tomaytoes I think tomahtoes, WebBusiness Magazine, April 1. 
http://www.cio.com/archive/webbusiness/040199/ nort.html

IBM (2001). Cost justifying ease of use: Complex solutions are problems. http://www.3ibm.co/ibm/easy/eou ext.nsf/Publish/23

Kalin, S. (1999). Mazed and confused, WebBusiness Magazine, April 1. http://www.cio.com/archive/webbusiness/040199/ use.html

Karat, C.M. (1997). cost-justifying usability engineering in the software life cycle, in T. Landauer \& P. Prabhu (Eds), Handbook of Human-Computer Interaction, Elsevier Science, Amsterdam.

Karat, C.M. (1990). Cost-benefit analysis of usability engineering techniques, Proceedings of the Human Factors Society $34^{t h}$. Annual Meeting, Volume 2, Orlando.

Lindgaard, G. (1992). Evaluating user interfaces in context: The ecological value of time-and-motion studies, Applied Ergonomics, 23, (2),105-114.

Lindgaard, G. (1994). Usability testing and system evaluation: A guide for designing useful computer systems, Chapman \& Hall, London.

Lindgaard, G. (1995). Cementing human factors into product design: Moving beyond policies, Proc. 15th. International Symposium on Human Factors in Telecommunications, Melbourne, 361-371.

Maguire, M. (1997). RESPECT: User Requirements Framework Handbook, HUSAT Research Institute.

Pressman, R. S. (1992). Software Engineering:A Practitioner's Approach, McGraw Hill, New York.

Rubin, J. (2002). What business are you in? The strategic role of usability professionals in the "New Economy" world, User Experience, Winter, 411.

Souza, R. ( 2001). Get ROI from design, The Forrester Report, June. Available from http://www.forrester.com

Stolovitch, H.D. \& Maurice, J.-G.(2001). Calculating your ROI: Calculating Return On Investment in human performance interventions and the increased value of human capital, http://www.creativityatwork.com

Vredenburg, K., Isensee, S., Righi, C., (2001). User-Centered Design: an Integrated Appraoch, Prentice Hall PTR, Upper Saddle River, NJ.

Wiklund, M.E.,(1994). Usability In Practice: How Companies Develop User-Friendly Products, Academic Press, Boston. 